\title{
Surrogate Endpoint
}

National Cancer Institute

\section{Source}

National Cancer Institute. Surrogate Endpoint. NCI Thesaurus. Code C68772.

A biomarker that is intended to substitute for a clinical endpoint. A surrog ate endpoint is expected to predict clinical benefit (or harm or lack of benefit or harm) based on epidemiologic, therapeutic, pathophysiologic, or other scientific evidence. The term surrogate endpoint applies primarily to endpoints in therapeutic intervention trials; however, it may sometimes apply in natural history or epidemiologic studies. The use of biomarkers as surrogate endpoints in a clinical trial requires the specification of the clinical endpoints that are being substituted, class of therapeutic intervention being applied, and characteristics of population and disease state in which the substitution is being made. 